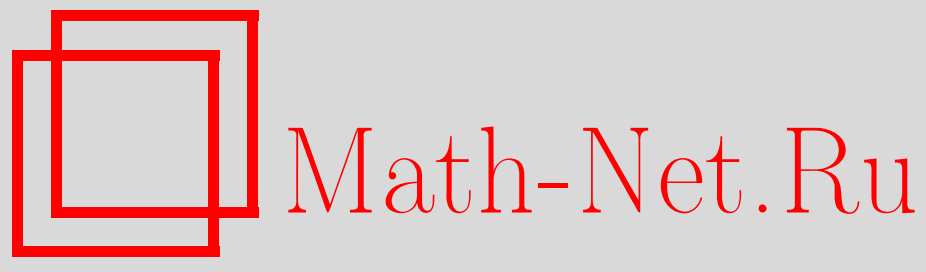

О. И. Мохов, Лиувиллева каноническая форма согласованных нелокальных скобок Пуассона гидродинамического типа и интегрируемые иерархии, Функи. анализ и его прил., 2003, том 37, выпуск 2, 28-40

DOI: https://doi.org/10.4213/faa146

Использование Общероссийского математического портала Math-Net.Ru подразумевает, что вы прочитали и согласны с пользовательским соглашением

http://www . mathnet.ru/rus/agreement

Параметры загрузки:

IP : 35.173 .219 .149

26 апреля 2023 г., 05:34:51

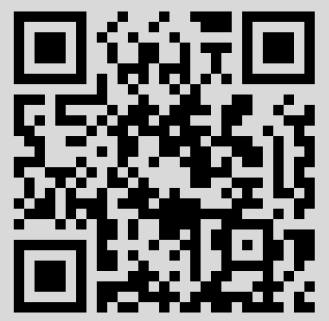




\title{
Лиувиллева каноническая форма согласованных нелокальных скобок Пуассона гидродинамического типа и интегрируемые иерархии*
}

\author{
(c) 2003. О. И. Мохов
}

\section{§1. Введение}

В настоящей работе мы, прежде всего, решаем задачу о приведении к наиболее простому и удобному для наших дальнейших целей «каноническому» виду произвольной пары согласованных нелокальных скобок Пуассона гидродинамического типа, порождаемых метриками постоянной римановой кривизны (согласованных скобок Мохова-Ферапонтова [1]), для того чтобы эффективно построить отвечающие любым этим парам согласованных скобок Пуассона интегрируемые иерархии. Как показано в $[2,3]$ (см. также [4]), согласованные скобки МоховаФерапонтова описываются совместной нелинейной системой уравнений, интегрируемой методом обратной задачи рассеяния (в случае согласованных скобок Дубровина-Новикова [5], т. е. согласованных локальных скобок Пуассона гидродинамического типа, соответствующая система проинтегрирована ранее в [6-8], см. также [4]), но задача эффективного построения в этом случае соответствующих интегрируемых иерархий, что является главной целью данной работы, требует иного подхода к описанию этих согласованных скобок. В данной статье по любому решению интегрируемой системы уравнений, описывающей рассматриваемые согласованные скобки, т.е. для любой пары этих согласованных скобок, построены в явном виде интегрируемые бигамильтоновы системы гидродинамического типа, обладающие этой парой согласованных нелокальных скобок Пуассона гидродинамического типа. В случае согласованных скобок ДубровинаНовикова [5] эта задача была рассмотрена и полностью решена ранее в работах автора $[9,10]$.

В [1] были введены и изучены нелокальные скобки Пуассона гидродинамического типа, имеющие следующий вид (скобки Мохова-Ферапонтова):

$$
\{I, J\}=\int \frac{\delta I}{\delta u^{i}(x)}\left(g^{i j}(u(x)) \frac{d}{d x}+b_{k}^{i j}(u(x)) u_{x}^{k}+K u_{x}^{i}\left(\frac{d}{d x}\right)^{-1} u_{x}^{j}\right) \frac{\delta J}{\delta u^{j}(x)} d x,
$$

где $I[u]$ и $J[u]-$ произвольные функционалы на пространстве функций (полей) $u^{i}(x), 1 \leqslant i \leqslant N$, одной независимой переменной $x, u=\left(u^{1}, \ldots, u^{N}\right)-$ локальные координаты на некотором заданном гладком $N$-мерном многообразии $M$, коэффициенты $g^{i j}(u)$ и $b_{k}^{i j}(u)$ скобки $(1.1)$ - гладкие функции локальных координат и $K-$ произвольная константа. Вид скобки (1.1) инвариантен отно-

*Работа выполнена при финансовой поддержке Фонда Александра фон Гумбольдта (Германия), а также Российского фонда фундаментальных исследований (грант №02-01-00803) и фонда INTAS (грант №99-1782). 
сительно локальных замен координат. Скобка вида (1.1) называется невырожденной, если $\operatorname{det}\left(g^{i j}(u)\right) \not \equiv 0$. Если $\operatorname{det}\left(g^{i j}(u)\right) \not \equiv 0$, то скобка (1.1) является скобкой Пуассона тогда и только тогда, когда $g^{i j}(u)-$ произвольная псевдориманова контравариантная метрика постоянной римановой кривизны $K$ и $b_{k}^{i j}(u)=-g^{i s}(u) \Gamma_{s k}^{j}(u)$, где $\Gamma_{s k}^{j}(u)$ - риманова связность, порождаемая метрикой $g^{i j}(u)$ (связность Леви-Чивиты) [1] (отметим, что при локальных заменах координат коэффициенты $g^{i j}(u)$ и $b_{k}^{i j}(u)$ скобки (1.1) преобразуются как соответствующие дифференциально-геометрические объекты - контравариантная метрика $g^{i j}(u)$ и связность $b_{k}^{i j}(u)=-g^{i s}(u) \Gamma_{s k}^{j}(u)$ соответственно, а $K-$ ин- $^{-}$ вариант). При $K=0$ получаем локальные скобки Пуассона гидродинамического типа (скобки Дубровина-Новикова [5]).

Напомним, что скобки Пуассона называются согласованными, если их произвольная линейная комбинация также является скобкой Пуассона (Магри [11]).

\section{§2. Согласованные нелокальные скобки Пуассона гидродинамического типа}

ЛЕмма 2.1. В задаче об описании произвольной пары согласованньх нелокальных скобок Пуассона вида (1.1) одну из этих двух скобок Пуассона всегда можно считать локальной без ограничения общности.

Действительно, если две согласованные нелокальные скобки Пуассона вида (1.1) $\{I, J\}_{0}$ (с соответствующей константой $K_{0}$ в нелокальной части) и $\{I, J\}_{1}$ (с константой $K_{1}$ ) линейно независимы, то в пучке этих скобок Пуассона, т. е. среди скобок Пуассона $\lambda_{0}\{I, J\}_{0}+\lambda_{1}\{I, J\}_{1}$, где $\lambda_{0}$ и $\lambda_{1}-$ произвольные константы, обязательно есть ненулевая локальная скобка Пуассона $\{I, J\}=\lambda_{0}^{\prime}\{I, J\}_{0}+\lambda_{1}^{\prime}\{I, J\}_{1}$, где $\lambda_{0}^{\prime}, \lambda_{1}^{\prime}$ - произвольные константы, удовлетворяющие соотношению $\lambda_{0}^{\prime} K_{0}+\lambda_{1}^{\prime} K_{1}=0$, которую и можно взять в качестве одной из образующих всего рассматриваемого пучка согласованных скобок Пуассона (очевидно, что если эта локальная скобка Пуассона $\{I, J\}$ нулевая, то скобки Пуассона $\{I, J\}_{0}$ и $\{I, J\}_{1}$ являются линейно зависимыми: $\left.\lambda_{0}^{\prime}\{I, J\}_{0}+\lambda_{1}^{\prime}\{I, J\}_{1} \equiv 0\right)$.

Рассмотрим задачу о согласованности нелокальной и локальной скобок Пуассона гидродинамического типа

$$
\{I, J\}_{1}=\int \frac{\delta I}{\delta u^{i}(x)}\left(g_{1}^{i j}(u(x)) \frac{d}{d x}+b_{1, k}^{i j}(u(x)) u_{x}^{k}+K_{1} u_{x}^{i}\left(\frac{d}{d x}\right)^{-1} u_{x}^{j}\right) \frac{\delta J}{\delta u^{j}(x)} d x
$$

и

$$
\{I, J\}_{2}=\int \frac{\delta I}{\delta u^{i}(x)}\left(g_{2}^{i j}(u(x)) \frac{d}{d x}+b_{2, k}^{i j}(u(x)) u_{x}^{k}\right) \frac{\delta J}{\delta u^{j}(x)} d x,
$$

т. е. условие, что при всех значениях константы $\lambda$ скобка

$$
\{I, J\}=\{I, J\}_{1}+\lambda\{I, J\}_{2}
$$

является скобкой Пуассона (таким образом, формула (2.3) определяет пучок согласованных скобок Пуассона).

Мы далее предполагаем, что локальная скобка $\{I, J\}_{2}$ является невырожденной, т. е. $\operatorname{det} g_{2}^{i j}(u) \not \equiv 0$, но не налагаем никаких дополнительных условий на 
скобку $\{I, J\}_{1}$, т. е., вообще говоря, она может быть и вырожденной. Скобка (2.3) может быть вырожденной; поэтому нам потребуются общие соотношения на коэффициенты скобки вида (1.1), эквивалентные условию, что эта скобка является скобкой Пуассона. Эти общие соотношения (без предположения невырожденности) были получены в работе автора [12] (см. также [13]):

$$
\begin{gathered}
g^{i j}(u)=g^{j i}(u), \\
\frac{\partial g^{i j}}{\partial u^{k}}=b_{k}^{i j}(u)+b_{k}^{j i}(u), \\
g^{i s}(u) b_{s}^{j r}(u)=g^{j s}(u) b_{s}^{i r}(u), \\
g^{i s}(u)\left(\frac{\partial b_{s}^{j r}}{\partial u^{k}}-\frac{\partial b_{k}^{j r}}{\partial u^{s}}\right)+b_{s}^{i j}(u) b_{k}^{s r}(u)-b_{s}^{i r}(u) b_{k}^{s j}(u)=K\left(g^{i r}(u) \delta_{k}^{j}-g^{i j}(u) \delta_{k}^{r}\right), \\
\sum_{(i, j, r)}\left[b_{p}^{s i}(u)\left(\frac{\partial b_{k}^{j r}}{\partial u^{s}}-\frac{\partial b_{s}^{j r}}{\partial u^{k}}\right)+K\left(b_{k}^{i j}(u)-b_{k}^{j i}(u)\right) \delta_{p}^{r}\right. \\
\left.+b_{k}^{s i}(u)\left(\frac{\partial b_{p}^{j r}}{\partial u^{s}}-\frac{\partial b_{s}^{j r}}{\partial u^{p}}\right)+K\left(b_{p}^{i j}(u)-b_{p}^{j i}(u)\right) \delta_{k}^{r}\right]=0
\end{gathered}
$$

где $\sum_{(i, j, r)}$ означает суммирование по всем циклическим перестановкам индексов $i, j, r$.

\section{§3. Каноническая форма согласованных пар скобок}

По теореме Дубровина-Новикова [5] для невырожденной локальной скобки Пуассона гидродинамического типа $\{I, J\}_{2}$ всегда существуют локальные координаты $u^{1}, \ldots, u^{N}$ (плоские координаты метрики $g_{2}^{i j}(u)$ ), в которых эта скобка постоянна, т.е. $g_{2}^{i j}(u)=\eta^{i j}=\mathrm{const}, b_{2, k}^{i j}(u)=\Gamma_{2, j k}^{i}(u)=0$. Таким образом, мы можем выбрать плоские координаты метрики $g_{2}^{i j}(u)$ и в дальнейшем считать, что скобка Пуассона $\{I, J\}_{2}$ постоянна и имеет вид

$$
\{I, J\}_{2}=\int \frac{\delta I}{\delta u^{i}(x)} \eta^{i j} \frac{d}{d x} \frac{\delta J}{\delta u^{j}(x)} d x,
$$

где $\eta^{i j}=\eta^{j i}, \eta^{i j}=$ const, $\operatorname{det} \eta^{i j} \neq 0$. В дальнейшем мы будем также использовать в рассматриваемых плоских координатах ковариантную метрику $\eta_{i j}$, обратную к контравариантной метрике $\eta^{i j}, \eta^{i s} \eta_{s j}=\delta_{j}^{i}$.

Теорема 3.1. Произвольная нелокальная скобка Пуассона $\{I, J\}_{1}$ вида (2.1) (возможно, вырожденная) согласована с постоянной скобкой Пуассона (3.1) тогда и только тогда, когда она имеет вид

$$
\begin{aligned}
\{I, J\}_{1}=\int \frac{\delta I}{\delta u^{i}(x)}\left(\left[\eta^{i s} \frac{\partial H^{j}}{\partial u^{s}}+\eta^{j s} \frac{\partial H^{i}}{\partial u^{s}}-K_{1} u^{i} u^{j}\right] \frac{d}{d x}\right. \\
\left.+\left[\eta^{i s} \frac{\partial^{2} H^{j}}{\partial u^{s} \partial u^{k}}-K_{1} \delta_{k}^{i} u^{j}\right] u_{x}^{k}+K_{1} u_{x}^{i}\left(\frac{d}{d x}\right)^{-1} u_{x}^{j}\right) \frac{\delta J}{\delta u^{j}(x)} d x,
\end{aligned}
$$

где $H^{i}(u), 1 \leqslant i \leqslant N,-$ гладкие функции, определенные в некоторой области локальных координат. 
В плоском случае согласованных скобок Дубровина-Новикова $\left(K_{1}=0\right)$ соответствующее утверждение было сформулировано автором в $[14,15]$ (см. также условия на плоские пучки метрик в [16]).

ДокАЗАТЕЛЬСтво. Из соотношений (2.4)-(2.8) следует, что в рассматриваемых локальных координатах условия согласованности скобок Пуассона $\{I, J\}_{1}$ и $\{I, J\}_{2}$ или, другими словами, условия, что скобка

$$
\begin{aligned}
\{I, J\}=\int \frac{\delta I}{\delta u^{i}(x)}\left(\left(g_{1}^{i j}(u(x))\right.\right. & \left.+\lambda \eta^{i j}\right) \frac{d}{d x} \\
& \left.+b_{1, k}^{i j}(u(x)) u_{x}^{k}+K_{1} u_{x}^{i}\left(\frac{d}{d x}\right)^{-1} u_{x}^{j}\right) \frac{\delta J}{\delta u^{j}(x)} d x
\end{aligned}
$$

является скобкой Пуассона для всех значений параметра $\lambda$, имеют вид

$$
\begin{aligned}
\eta^{i s} b_{1, s}^{j r}(u) & =\eta^{j s} b_{1, s}^{i r}(u), \\
\frac{\partial b_{1, s}^{j r}}{\partial u^{k}}-\frac{\partial b_{1, k}^{j r}}{\partial u^{s}} & =K_{1}\left(\delta_{s}^{r} \delta_{k}^{j}-\delta_{s}^{j} \delta_{k}^{r}\right) .
\end{aligned}
$$

Определим функции $A_{k}^{i j}(u)$ соотношениями

$$
A_{k}^{i j}(u)=b_{1, k}^{i j}(u)-K_{1} \delta_{k}^{j} u^{i} .
$$

Из формулы (3.5) следует, что

$$
\frac{\partial A_{s}^{j r}}{\partial u^{k}}-\frac{\partial A_{k}^{j r}}{\partial u^{s}}=0
$$

т. е. по лемме Пуанкаре локально существуют функции $P^{i j}(u)$, такие, что

$$
A_{k}^{i j}(u)=\frac{\partial P^{i j}}{\partial u^{k}}
$$

и из (3.6) находим необходимое выражение для коэффициента $b_{1, k}^{i j}(u)$ :

$$
b_{1, k}^{i j}(u)=\frac{\partial P^{i j}}{\partial u^{k}}+K_{1} \delta_{k}^{j} u^{i} .
$$

Найдем выражение для метрики $g_{1}^{i j}(u)$. Из соотношения $(2.5)$ для скобки Пуассона $\{I, J\}_{1}$ получаем

$$
\frac{\partial g_{1}^{i j}}{\partial u^{k}}=\frac{\partial P^{i j}}{\partial u^{k}}+\frac{\partial P^{j i}}{\partial u^{k}}+K_{1} \delta_{k}^{j} u^{i}+K_{1} \delta_{k}^{i} u^{j}
$$

и, следовательно, учитывая соотношение (2.4),

$$
g_{1}^{i j}(u)=P^{i j}(u)+P^{j i}(u)+K_{1} u^{i} u^{j}+c^{i j}, \quad c^{i j}=\text { const, } c^{i j}=c^{j i} .
$$

Таким образом, доказано, что коэффициенты скобки Пуассона $\{I, J\}_{1}$ имеют так называемый лиувиллев вид (подробнее о важном свойстве лиувиллевости см. $§ 5)$ :

$$
\begin{gathered}
g_{1}^{i j}(u)=R^{i j}(u)+R^{j i}(u)+K_{1} u^{i} u^{j}, \\
b_{1, k}^{i j}(u)=\frac{\partial R^{i j}}{\partial u^{k}}+K_{1} u^{i} \delta_{k}^{j},
\end{gathered}
$$


где

$$
R^{i j}(u)=P^{i j}(u)+\frac{1}{2} c^{i j} .
$$

Кроме того, из соотношения (3.4) дополнительно получаем

$$
\eta_{p j} b_{1, l}^{j r}(u)=\eta_{l i} b_{1, p}^{i r}(u)
$$

T. e.

$$
\frac{\partial\left(\eta_{p s} R^{s r}(u)\right)}{\partial u^{l}}+K_{1} \eta_{p s} u^{s} \delta_{l}^{r}=\frac{\partial\left(\eta_{l s} R^{s r}(u)\right)}{\partial u^{p}}+K_{1} \eta_{l s} u^{s} \delta_{p}^{r} .
$$

Последняя формула эквивалентна соотношению

$$
\frac{\partial\left(\eta_{p s} R^{s r}(u)+K_{1} \eta_{p s} u^{s} u^{r}\right)}{\partial u^{l}}=\frac{\partial\left(\eta_{l s} R^{s r}(u)+K_{1} \eta_{l s} u^{s} u^{r}\right)}{\partial u^{p}} .
$$

Следовательно, по лемме Пуанкаре локально существуют функции $H^{r}(u), 1 \leqslant$ $r \leqslant N$, такие, что

Таким образом, доказано, что

$$
\eta_{p s} R^{s r}(u)+K_{1} \eta_{p s} u^{s} u^{r}=\frac{\partial H^{r}}{\partial u^{p}} .
$$

$$
R^{s r}(u)=\eta^{s p} \frac{\partial H^{r}}{\partial u^{p}}-K_{1} u^{s} u^{r}
$$

а коэффициенты скобки Пуассона $\{I, J\}_{1}$ имеют вид

$$
\begin{gathered}
g_{1}^{i j}(u)=\eta^{i s} \frac{\partial H^{j}}{\partial u^{s}}+\eta^{j s} \frac{\partial H^{i}}{\partial u^{s}}-K_{1} u^{i} u^{j}, \\
b_{1, k}^{i j}(u)=\eta^{i s} \frac{\partial^{2} H^{j}}{\partial u^{s} \partial u^{k}}-K_{1} \delta_{k}^{i} u^{j} .
\end{gathered}
$$

Поскольку в этом случае, как легко проверить, все соотношения согласованности (3.4) и (3.5) выполнены, важная для всей нашей дальнейшей конструкции теорема 3.1 доказана.

\section{§4. Интегрируемые уравнения для канонических согласованных пар скобок}

ТЕорема 4.1. Нелокальная скобка вида (3.2) (возможно, вырожденная) является скобкой Пуассона тогда и только тогда, когда выполнены следующие уравнения:

$$
\begin{gathered}
\frac{\partial^{2} H^{i}}{\partial u^{k} \partial u^{s}} \eta^{s p} \frac{\partial^{2} H^{j}}{\partial u^{p} \partial u^{l}}=\frac{\partial^{2} H^{j}}{\partial u^{k} \partial u^{s}} \eta^{s p} \frac{\partial^{2} H^{i}}{\partial u^{p} \partial u^{l}} \\
\left(\eta^{i r} \frac{\partial H^{s}}{\partial u^{r}}+\eta^{s r} \frac{\partial H^{i}}{\partial u^{r}}-K_{1} u^{i} u^{s}\right) \eta^{j p} \frac{\partial^{2} H^{k}}{\partial u^{p} \partial u^{s}} \\
=\left(\eta^{j r} \frac{\partial H^{s}}{\partial u^{r}}+\eta^{s r} \frac{\partial H^{j}}{\partial u^{r}}-K_{1} u^{j} u^{s}\right) \eta^{i p} \frac{\partial^{2} H^{k}}{\partial u^{p} \partial u^{s}}
\end{gathered}
$$

В плоском случае $\left(K_{1}=0\right)$ соответствующая теорема получена автором в [15], где была сформулирована также и гипотеза об интегрируемости системы (4.1), (4.2) при $K_{1}=0$ методом обратной задачи рассеяния, доказанная впоследствии в работах автора $[4,6,7]$ (см. также [8], где найдена пара Лакса для этой системы 
в плоском случае). Соответствующие общие условия на плоские пучки метрик указаны в [16].

Для нелокальной скобки вида (3.2) соотношения $(2.4),(2.5),(2.8)$ выполняются тождественно. Соотношение $(2.7)$ в этом случае принимает вид

$$
b_{1, s}^{i j}(u) b_{1, k}^{s r}(u)=b_{1, s}^{i r}(u) b_{1, k}^{s j}(u),
$$

что дает уравнения

$$
\begin{aligned}
\left(\eta^{i p} \frac{\partial^{2} H^{j}}{\partial u^{p} \partial u^{s}}-K_{1} \delta_{s}^{i} u^{j}\right) & \left(\eta^{s l} \frac{\partial^{2} H^{r}}{\partial u^{l} \partial u^{k}}-K_{1} \delta_{k}^{s} u^{r}\right) \\
= & \left(\eta^{i p} \frac{\partial^{2} H^{r}}{\partial u^{p} \partial u^{s}}-K_{1} \delta_{s}^{i} u^{r}\right)\left(\eta^{s l} \frac{\partial^{2} H^{j}}{\partial u^{l} \partial u^{k}}-K_{1} \delta_{k}^{s} u^{j}\right),
\end{aligned}
$$

эквивалентные уравнениям (4.1).

Соотношение (2.6) дает уравнения

$$
\begin{aligned}
\left(\eta^{i p} \frac{\partial H^{s}}{\partial u^{p}}+\eta^{s p}\right. & \left.\frac{\partial H^{i}}{\partial u^{p}}-K_{1} u^{i} u^{s}\right)\left(\eta^{j l} \frac{\partial^{2} H^{r}}{\partial u^{l} \partial u^{s}}-K_{1} \delta_{s}^{j} u^{r}\right) \\
& =\left(\eta^{j p} \frac{\partial H^{s}}{\partial u^{p}}+\eta^{s p} \frac{\partial H^{j}}{\partial u^{p}}-K_{1} u^{j} u^{s}\right)\left(\eta^{i l} \frac{\partial^{2} H^{r}}{\partial u^{l} \partial u^{s}}-K_{1} \delta_{s}^{i} u^{r}\right),
\end{aligned}
$$

эквивалентные уравнениям (4.2).

Из теоремы 4.1 немедленно получаем следующее элементарное следствие.

СлеДСТвиЕ 4.1. Очевидно, ито любой набор $N$ линейных функиций $H^{i}(u)=$ $c_{k}^{i} u^{k}+c^{i}, c_{k}^{i}=$ const, $c^{i}=$ const, является тривиальным решением нелинейной системь уравнений (4.1), (4.2). Таким образом, скобка

$$
\begin{aligned}
\{I, J\}_{1}=\int \frac{\delta I}{\delta u^{i}(x)}\left(\left[\eta^{i s} c_{s}^{j}+\right.\right. & \left.\eta^{j s} c_{s}^{i}-K_{1} u^{i} u^{j}\right] \frac{d}{d x} \\
& \left.-K_{1} \delta_{k}^{i} u^{j} u_{x}^{k}+K_{1} u_{x}^{i}\left(\frac{d}{d x}\right)^{-1} u_{x}^{j}\right) \frac{\delta J}{\delta u^{j}(x)} d x
\end{aligned}
$$

является скобкой Пуассона при любых константах $c_{k}^{i}$. В частности, для любой симметричной постоянной матрицьь $\left(\mu^{i j}\right), \mu^{i j}=\mu^{j i}, \mu^{i j}=\mathrm{const,} \mathrm{кон-}$ травариантная метрика $\mu^{i j}-K u^{i} u^{j}$ всегда является метрикой постоянной кривизны $K$, если она невырожденна (при условии невырожденности матрицьь $\left(\mu^{i j}\right)$ эта метрика является, очевидно, невырожденной); при этом порождаемая этой метрикой связность Леви-Чивить определяется формулой $\Gamma_{k}^{i j}(u)=\left(\mu^{i s}-K u^{i} u^{s}\right) \Gamma_{s k}^{j}(u)=K \delta_{k}^{i} u^{j}$.

Рассмотрим, в частности, контравариантную метрику вида

$$
g^{i j}(u)=a^{i} \delta^{i j}-K u^{i} u^{j},
$$

где $a^{i}, 1 \leqslant i \leqslant N$, - произвольные ненулевые константы, так что метрика $g^{i j}(u)$ является невырожденной. Нетрудно доказать, что

$$
\operatorname{det}\left(g^{i j}(u)\right)=a^{1} \cdots a^{N}\left(1-K \sum_{s=1}^{N} \frac{\left(u^{s}\right)^{2}}{a^{s}}\right) .
$$


Ковариантная метрика $g_{i j}(u)$, обратная к контравариантной метрике $g^{i j}(u)$, $g^{i s}(u) g_{s j}(u)=\delta_{j}^{i}$, имеет вид

$$
g_{i j}(u)=\frac{1}{a^{i}} \delta_{i j}+\frac{K u^{i} u^{j}}{a^{i} a^{j}\left(1-K \sum_{s=1}^{N} \frac{\left(u^{s}\right)^{2}}{a^{s}}\right)}
$$

и является метрикой постоянной римановой кривизны $K$ при любых значениях ненулевых констант $a^{i}$. Любопытно, что рассматриваемые локальные координаты являются геодезическими в точке с координатами $(0, \ldots, 0)\left(u^{i}=0,1 \leqslant i \leqslant N\right)$, но не являются не только нормальными координатами Биркгофа для метрики, но даже и римановыми координатами, в которых, как известно, метрика имеет подобное разложение. Более важно, что эти локальные координаты являются, как будет показано в $\$ 5$, специальными лиувиллевыми координатами для соответствующей скобки Пуассона.

Отметим, что метрика $g^{i j}(u)$ является невырожденной тогда и только тогда, когда не более чем одна из $N+1$ констант $a^{i}, 1 \leqslant i \leqslant N$, и $K$ равна нулю. Укажем соответствующие формулы для интересного случая, когда любая одна из этих констант равна нулю. Случай $K=0$ тривиален. Если же $a^{m}=0$, то

$$
\operatorname{det}\left(g^{i j}(u)\right)=-K\left(\prod_{s \neq m} a^{s}\right)\left(u^{m}\right)^{2} .
$$

Компоненты ковариантной метрики $g_{i j}(u)$ постоянной римановой кривизны $K$ в этом случае имеют вид

$$
\begin{gathered}
g_{m m}(u)=-\frac{1}{K\left(u^{m}\right)^{2}}\left(1-K \sum_{s \neq m} \frac{\left(u^{s}\right)^{2}}{a^{s}}\right), \\
g_{i m}(u)=g_{m i}(u)=-\frac{1}{a^{i}} \frac{u^{i}}{u^{m}}, \quad i \neq m, \\
g_{i i}(u)=\frac{1}{a^{i}}, \quad i \neq m, \\
g_{i j}(u)=0, \quad i \neq j, \quad i \neq m, j \neq m .
\end{gathered}
$$

Все эти модели пространств постоянной кривизны играют важную роль в гамильтоновой теории систем гидродинамического типа. Скобки Мохова-Ферапонтова, задаваемые при $a^{i}=\varepsilon^{i}= \pm 1$ метриками (4.7) постоянной римановой кривизны $K$, названы в [17] каноническими. Такие канонические скобки естественно возникали также в приложениях в работе [18].

Теорема 4.2 [2,3]. Система нелинейных уравнений (4.1), (4.2) интегрируется методом обратной задачи рассеяния.

Отметим, что в $[2,3]$ получена и проинтегрирована система нелинейных уравнений, эквивалентная системе (4.1), (4.2) и тоже описывающая согласованные нелокальные скобки Пуассона вида (1.1), но в других локальных координатах, гораздо более удобных для интегрирования (в этих координатах метрики обеих согласованных скобок диагональны). Но локальные координаты, в которых 
метрики скобок диагонализируются, плохо приспособлены для эффективного построения интегрируемых иерархий, соответствующих рассматриваемым согласованным скобкам Пуассона. Для этой цели в данной работе развит другой подход. В плоском случае $\left(K_{1}=0\right)$ система $(4.1),(4.2)$ проинтегрирована в $[6,7]$.

\section{§5. Лиувиллевы и специальные лиувиллевы координаты}

ОПРЕДЕЛЕНИЕ 5.1. Локальные координаты $u=\left(u^{1}, \ldots, u^{N}\right)$ называются $л u y$ виллевыми для произвольной скобки Пуассона $\{I, J\}$, если функции (поля) $u^{i}(x)$ являются плотностями интегралов в инволюции относительно этой скобки, т. е.

$$
\left\{U^{i}, U^{j}\right\}=0, \quad 1 \leqslant i, j \leqslant N,
$$

где $U^{i}=\int u^{i}(x) d x, 1 \leqslant i \leqslant N$. В этом случае скобка Пуассона также называется лиувиллевой в этих координатах.

Лиувиллевы координаты естественно возникают и играют существенную роль в процедуре Дубровина-Новикова усреднения гамильтоновых уравнений [5]. Физические координаты, получаемые усреднением плотностей участвующих в процедуре Дубровина-Новикова $N$ инволютивных локальных интегралов изначальной гамильтоновой системы, всегда являются лиувиллевыми для соответствующей усредненной скобки. Это свойство и явилось мотивировкой определения лиувиллевых координат для локальных скобок Пуассона гидродинамического типа в [5]. Для общих слабо нелокальных скобок Пуассона гидродинамического типа (скобок Ферапонтова $[19,20])$ лиувиллевы координаты введены в [17].

Нелокальная скобка Пуассона гидродинамического типа (1.1) является лиувиллевой в локальных координатах $u=\left(u^{1}, \ldots, u^{N}\right)$ тогда и только тогда, когда существует матричная функция $\Phi^{i j}(u)$, такая, что

$$
b_{k}^{i j}(u)=\frac{\partial \Phi^{i j}}{\partial u^{k}}-K \delta_{k}^{i} u^{j} .
$$

В этом случае в силу соотношений (2.4), (2.5) метрика $g^{i j}(u)$ обязана иметь вид

$$
g^{i j}(u)=\Phi^{i j}(u)+\Phi^{j i}(u)-K u^{i} u^{j}
$$

(функцию $\Phi^{i j}(u)$ при этом можно подправить на произвольную постоянную матричную функцию $c^{i j}=$ const). Матричная функция $\Phi^{i j}(u)$ называется лиувиллевой функцией соответствующей лиувиллевой скобки Пуассона.

Таким образом, нелокальная скобка Пуассона (1.1) лиувиллева, если она имеет вид

$$
\begin{aligned}
\{I, J\}=\int \frac{\delta I}{\delta u^{i}(x)}( & \left(\Phi^{i j}(u)+\Phi^{j i}(u)-K u^{i} u^{j}\right) \frac{d}{d x} \\
& \left.+\left(\frac{\partial \Phi^{i j}}{\partial u^{k}}-K \delta_{k}^{i} u^{j}\right) u_{x}^{k}+K u_{x}^{i}\left(\frac{d}{d x}\right)^{-1} u_{x}^{j}\right) \frac{\delta J}{\delta u^{j}(x)} d x .
\end{aligned}
$$

Из теоремы 3.1 следует 
ТЕОРема 5.1. Плоские координаты произвольной невырожденной локальной скобки Пуассона гидродинамического типа $\{I, J\}_{2}$ всегда являются лиувиллевыми для любой нелокальной скобки Пуассона $\{I, J\}_{1}$ вида (1.1), согласованной с $\{I, J\}_{2}$. Более того, при этом соответствующая лиувиллева функция $\Phi^{i j}(u)$ всегда имеет специиальньй вид

$$
\Phi^{i j}(u)=\eta^{i s} \frac{\partial H^{j}}{\partial u^{s}} .
$$

ОПРЕДЕЛЕНИЕ 5.2. Локальные координаты $u=\left(u^{1}, \ldots, u^{N}\right)$ называются cneцииальными лиувиллевыми координатами $[15,21]$ для произвольной скобки Пуассона $\{I, J\}$, если существует ненулевая постоянная симметричная матрица $\left(\eta_{i j}\right)$, такая, что функции (поля) $u^{i}(x), 1 \leqslant i \leqslant N$, и $\eta_{i j} u^{i}(x) u^{j}(x)$ являются плотностями интегралов в инволюции относительно этой скобки, т. е.

$$
\left\{U^{i}, U^{j}\right\}=0, \quad 1 \leqslant i, j \leqslant N+1,
$$

где $U^{i}=\int u^{i}(x) d x, 1 \leqslant i \leqslant N, U^{N+1}=\int \eta_{i j} u^{i}(x) u^{j}(x) d x$. В этом случае скобка Пуассона также называется спещзиальной лиувиллевой в этих координатах.

Специальные лиувиллевы координаты были введены в работах автора $[15,21]$. Наиболее важным является случай невырожденной матрицы $\eta_{i j}$.

Теорема 5.2. Нелокальная скобка Пуассона вида (1.1) является специальной лиувиллевой в локальных координатах $u=\left(u^{1}, \ldots, u^{N}\right)$ тогда и только тогда, когда она лиувиллева со спещиальной лиувиллевой функцией $\Phi^{i j}(u)$, такой, что

$$
\eta_{k s} \Phi^{s j}(u)=\frac{\partial H^{j}}{\partial u^{k}}
$$

Для невырожденной матрицы $\left(\eta_{i j}\right)$ получаем в этом случае в точности нашу скобку (3.2) из канонической согласованной пары.

Таким образом, наша задача о согласованных нелокальных скобках Пуассона гидродинамического типа эквивалентна задаче классификации специальных лиувиллевых координат для нелокальных скобок Пуассона гидродинамического типа.

Теорема 5.3. Произвольная нелокальная скобка Пуассона гидродинамического типа вида (1.1) согласована с постоянной скобкой Пуассона (3.1) тогда и только тогда, когда функции $u^{i}(x), 1 \leqslant i \leqslant N, u \eta_{i j} u^{i}(x) u^{j}(x), \eta^{i s} \eta_{s j}=\delta_{j}^{i}$, являются плотностями интегралов в инволюции относительно скобки Пуассона (1.1).

Отметим, что $u^{i}(x), 1 \leqslant i \leqslant N$, - плотности аннуляторов скобки (3.1), а $\frac{1}{2} \eta_{i j} u^{i}(x) u^{j}(x)-$ плотность импульса скобки (3.1).

Теорема 5.4. Произвольная нелокальная скобка Пуассона гидродинамического типа вида (1.1) согласована с произвольной невырожденной локальной скобкой Пуассона (2.2) тогда и только тогда, когда $N$ аннуляторов и импульс скобки (2.2) являются интегралами в инволющии относительно скобки Пуассона (1.1).

В плоском случае $K=0$ все утверждения данного параграфа были доказаны ранее в работах автора $[14,15,21]$. 


\section{§6. Интегрируемые бигамильтоновы системы гидродинамического типа}

Рассмотрим произвольную пару согласованных нелокальных гамильтоновых операторов гидродинамического типа $P_{1}^{i j}$ и $P_{2}^{i j}$, порождаемых метриками постоянной римановой кривизны. Как показано выше в лемме 2.1, один из этих операторов, скажем $P_{2}^{i j}$, можно считать локальным без ограничения общности. Если локальный гамильтонов оператор $P_{2}^{i j}$ является невырожденным, то из теоремы 3.1 следует, что локальной заменой координат пара согласованных гамильтоновых операторов $P_{1}^{i j}$ и $P_{2}^{i j}$ приводится к следующему каноническому виду:

$$
\begin{aligned}
P_{2}^{i j}[v(x)]= & \eta^{i j} \frac{d}{d x} \\
P_{1}^{i j}[v(x)]= & \left(\eta^{i s} \frac{\partial h^{j}}{\partial v^{s}}+\eta^{j s} \frac{\partial h^{i}}{\partial v^{s}}-K v^{i} v^{j}\right) \frac{d}{d x} \\
& +\left(\eta^{i s} \frac{\partial^{2} h^{j}}{\partial v^{s} \partial v^{k}}-K \delta_{k}^{i} v^{j}\right) v_{x}^{k}+K v_{x}^{i}\left(\frac{d}{d x}\right)^{-1} v_{x}^{j},
\end{aligned}
$$

где $\left(\eta^{i j}\right)$ - произвольная невырожденная постоянная симметричная матрица, т. е. $\operatorname{det}\left(\eta^{i j}\right) \neq 0, \eta^{i j}=\mathrm{const}, \eta^{i j}=\eta^{j i} ; K-$ произвольная константа; $h^{i}(v)$, $1 \leqslant i \leqslant N$, - гладкие функции, заданные в некоторой области локальных координат, такие, что оператор (6.2) является гамильтоновым, т. е. функции $h^{i}(v)$ удовлетворяют интегрируемым уравнениям (4.1), (4.2) (см. теоремы 4.1 и 4.2 выше).

ЗАмЕчАНИЕ 6.1. Очевидно, что здесь всегда можно считать, что $\eta^{i j}=\varepsilon^{i} \delta^{i j}$, $\varepsilon^{i}=1$ при $i \leqslant p, \varepsilon^{i}=-1$ при $i>p$, где $p-$ положительный индекс инерции метрики, $0 \leqslant p \leqslant N$, и, кроме того, необходимо классифицировать гамильтоновы операторы (6.2) относительно действия группы движений соответствующего $N$-мерного псевдоевклидова пространства $\mathbb{R}_{p}^{N}$. Это нетрудно сделать, но для наших целей достаточно (и удобнее) пользоваться указанным выше представлением канонической согласованной пары («условно каноническим» представлением).

Рассмотрим оператор рекурсии, задаваемый каноническими согласованными гамильтоновыми операторами (6.1), (6.2),

$$
\begin{aligned}
R_{l}^{i}[v(x)]= & {\left[P_{1}[v(x)]\left(P_{2}[v(x)]\right)^{-1}\right]_{l}^{i} } \\
= & \left(\left(\eta^{i s} \frac{\partial h^{j}}{\partial v^{s}}+\eta^{j s} \frac{\partial h^{i}}{\partial v^{s}}-K v^{i} v^{j}\right) \frac{d}{d x}\right. \\
& \left.+\left(\eta^{i s} \frac{\partial^{2} h^{j}}{\partial v^{s} \partial v^{k}}-K \delta_{k}^{i} v^{j}\right) v_{x}^{k}+K v_{x}^{i}\left(\frac{d}{d x}\right)^{-1} v_{x}^{j}\right) \eta_{j l}\left(\frac{d}{d x}\right)^{-1}
\end{aligned}
$$

(об операторах рекурсии, задаваемых парами согласованных гамильтоновых операторов, см. [13,22-26]).

Применим полученный нами оператор рекурсии (6.3) к системе трансляций по $x$, т. е. системе гидродинамического типа

$$
v_{t}^{i}=v_{x}^{i}
$$


которая, очевидно, является гамильтоновой с гамильтоновым оператором (6.1):

$$
v_{t}^{i}=v_{x}^{i} \equiv \eta^{i j} \frac{d}{d x} \frac{\delta H}{\delta v^{j}(x)}, \quad H=\frac{1}{2} \int \eta_{j l} v^{j}(x) v^{l}(x) d x .
$$

Любая система из иерархии

$$
v_{t_{n}}^{i}=\left(R^{n}\right)_{j}^{i} v_{x}^{j}, \quad n \in \mathbb{Z},
$$

является мультигамильтоновой интегрируемой системой.

В частности, интегрируемой является любая система вида

$$
v_{t_{1}}^{i}=R_{j}^{i} v_{x}^{j}
$$

т. е. система гидродинамического типа

$$
\begin{aligned}
v_{t_{1}}^{i}= & \left(\left(\eta^{i s} \frac{\partial h^{j}}{\partial v^{s}}+\eta^{j s} \frac{\partial h^{i}}{\partial v^{s}}-K v^{i} v^{j}\right) \frac{d}{d x}\right. \\
& \left.+\left(\eta^{i s} \frac{\partial^{2} h^{j}}{\partial v^{s} \partial v^{k}}-K \delta_{k}^{i} v^{j}\right) v_{x}^{k}+K v_{x}^{i}\left(\frac{d}{d x}\right)^{-1} v_{x}^{j}\right) \eta_{j l} v^{l} \\
\equiv & \left(\eta^{i s} \frac{\partial h^{j}}{\partial v^{s}} \eta_{j k}+\frac{\partial h^{i}}{\partial v^{k}}+\eta^{i s} \eta_{j l} \frac{\partial^{2} h^{j}}{\partial v^{s} \partial v^{k}} v^{l}-K \eta_{s k} v^{i} v^{s}-\frac{K}{2} \delta_{k}^{i} \eta_{s l} v^{s} v^{l}\right) v_{x}^{k} \\
\equiv & \left(h^{i}(v)+\eta^{i s} \frac{\partial h^{j}}{\partial v^{s}} \eta_{j l} v^{l}-\frac{K}{2} \eta_{s k} v^{i} v^{s} v^{k}\right)_{x}
\end{aligned}
$$

где $h^{i}(v), 1 \leqslant i \leqslant N,-$ произвольное решение интегрируемой системы (4.1), $(4.2)$.

Эта система гидродинамического типа является бигамильтоновой с парой канонических согласованных гамильтоновых операторов (6.1), (6.2):

$$
\begin{gathered}
v_{t_{1}}^{i}=\left(\left(\eta^{i s} \frac{\partial h^{j}}{\partial v^{s}}+\eta^{j s} \frac{\partial h^{i}}{\partial v^{s}}-K v^{i} v^{j}\right) \frac{d}{d x}\right. \\
\left.+\left(\eta^{i s} \frac{\partial^{2} h^{j}}{\partial v^{s} \partial v^{k}}-K \delta_{k}^{i} v^{j}\right) v_{x}^{k}+K v_{x}^{i}\left(\frac{d}{d x}\right)^{-1} v_{x}^{j}\right) \frac{\delta H_{1}}{\delta v^{j}(x)} \\
H_{1}=\frac{1}{2} \int \eta_{j l} v^{j}(x) v^{l}(x) d x \\
v_{t_{1}}^{i}=\eta^{i j} \frac{d}{d x} \frac{\delta H_{2}}{\delta v^{j}(x)}, \\
H_{2}=\int\left(\eta_{j k} h^{k}(v(x)) v^{j}(x)-\frac{K}{8} \eta_{j k} \eta_{s l} v^{j}(x) v^{k}(x) v^{s}(x) v^{l}(x)\right) d x
\end{gathered}
$$

Следующей системой в иерархии (6.6) (при $n=2$ ) является интегрируемая система гидродинамического типа

$$
\begin{aligned}
v_{t_{2}}^{i}=\left(\left(\eta^{i s} \frac{\partial h^{j}}{\partial v^{s}}+\eta^{j s}\right.\right. & \left.\frac{\partial h^{i}}{\partial v^{s}}-K v^{i} v^{j}\right) \frac{d}{d x}+\left(\eta^{i s} \frac{\partial^{2} h^{j}}{\partial v^{s} \partial v^{k}}-K \delta_{k}^{i} v^{j}\right) v_{x}^{k} \\
& \left.+K v_{x}^{i}\left(\frac{d}{d x}\right)^{-1} v_{x}^{j}\right) \eta_{j l}\left(h^{l}(v)+\eta^{l p} \frac{\partial h^{r}}{\partial v^{p}} \eta_{r q} v^{q}-\frac{K}{2} \eta_{p r} v^{l} v^{p} v^{r}\right)
\end{aligned}
$$




$$
\begin{gathered}
\equiv\left(( \eta ^ { i s } \frac { \partial h ^ { j } } { \partial v ^ { s } } + \eta ^ { j s } \frac { \partial h ^ { i } } { \partial v ^ { s } } - K v ^ { i } v ^ { j } ) \left(\eta_{j l} \frac{\partial h^{l}}{\partial v^{k}}+\eta_{r k} \frac{\partial h^{r}}{\partial v^{j}}+\eta_{r q} v^{q} \frac{\partial^{2} h^{r}}{\partial v^{j} \partial v^{k}}\right.\right. \\
\left.\quad-K \eta_{j l} \eta_{p k} v^{l} v^{p}-\frac{K}{2} \eta_{j k} \eta_{p l} v^{l} v^{p}\right) \\
+\left(\eta^{i s} \frac{\partial^{2} h^{j}}{\partial v^{s} \partial v^{k}}-K \delta_{k}^{i} v^{j}\right)\left(\eta_{j l} h^{l}(v)+\eta_{r q} v^{q} \frac{\partial h^{r}}{\partial v^{j}}-\frac{K}{2} \eta_{j l} \eta_{p r} v^{l} v^{p} v^{r}\right) \\
\left.+K \delta_{k}^{i}\left(\eta_{j l} h^{l}(v) v^{j}-\frac{K}{8} \eta_{j l} \eta_{p r} v^{j} v^{l} v^{p} v^{r}\right)\right) v_{x}^{k}
\end{gathered}
$$

Даже тривиальные, линейные по полям $v^{i}(x)$, решения системы $(4.1),(4.2)$ порождают нетривиальные интегрируемые системы гидродинамического типа. Все представленные в данной работе результаты обобщаются прямым образом на случай общих нелокальных скобок Пуассона гидродинамического типа (скобок Ферапонтова $[19,20])$, хотя формулы становятся значительно более громоздкими и менее эффективными. Эти результаты будут опубликованы в другой работе.

\section{ЛИТЕРАТУРА}

1. Мохов О. И., Ферапонтов E. В. О нелокальных гамильтоновых операторах гидродинамического типа, связанных с метриками постоянной кривизны. УМН, 45, вып. 3, 191-192 (1990).

2. Мохов О. И. Согласованные метрики постоянной римановой кривизны: локальная геометрия, нелинейные уравнения и интегрируемость. Функц. анализ и его прил., 36, вып. 3, 36-47 (2002); arXiv: math.DG/0201280 (2002).

3. Мохов О. И. Пара Лакса для неособых пучков метрик постоянной римановой кривизны. УМН, 57, вып. 3, 155-156 (2002).

4. Мохов О. И. Согласованные и почти согласованные псевдоримановы метрики. Функц. анализ и его прил., 35, вып. 2, 24-36 (2001); arXiv: math.DG/0005051 (2000).

5. Дубровин Б. А., Новиков С. П. Гамильтонов формализм одномерных систем гидродинамического типа и метод усреднения Боголюбова-Уизема. ДАН СССР, 270, № 4, 781-785 (1983).

6. Мохов О. И. Об интегрируемости уравнений для неособых пар согласованных плоских метрик. Теор. матем. физ., 130, вып. 2, 233-250 (2002); arXiv: math.DG/0005081 (2000).

7. Мохов О. И. Плоские пучки метрик и интегрируемые редукции уравнений Ламе. УМН, 56, вып. 2, 221-222 (2001).

8. Ferapontov E. V. Compatible Poisson brackets of hydrodynamic type. J. Phys. A, 34, 2377-2388 (2001); arXiv: math.DG/0005221 (2000).

9. Мохов О. И. Согласованные гамильтоновы операторы Дубровина-Новикова, производная Ли и интегрируемые системы гидродинамического типа. Труды Международной конференции «Нелинейные эволюционные уравнения и динамические системы», Кембридж (Англия), 24-30 июля 2001 г., Теор. матем. физ., 133, вып. 2, 279-288 (2002); arXiv: math.DG/0201281 (2002).

10. Мохов О. И. Интегрируемые бигамильтоновы системы гидродинамического типа. УМН, 57, вып. 1, 157-158 (2002).

11. Magri F. A simple model of the integrable Hamiltonian equation. J. Math. Phys., 19, No. 5, 1156-1162 (1978).

12. Mokhov O. I. Hamiltonian systems of hydrodynamic type and constant curvature metrics. Phys. Letters. A, 166, Nos. 3-4, 215-216 (1992). 
13. Мохов О. И. Симплектические и пуассоновы структуры на пространствах петель гладких многообразий и интегрируемые системы. УМН, 53, вып. 3, 85-192 (1998).

14. Мохов О. И. О согласованных пуассоновых структурах гидродинамического типа. УМН, 52, вып. 6, 171-172 (1997).

15. Мохов О. И. Согласованные пуассоновы структуры гидродинамического типа и уравнения ассоциативности. Труды МИРАН, 225, 284-300 (1999).

16. Dubrovin B. Geometry of 2D topological field theories. In: Lect. Notes in Math., Vol. 1620, 1996, pp. 120-348; arXiv: hep-th/9407018 (1994).

17. Maltsev A. Ya., Novikov S. P. On the local systems Hamiltonian in the weakly nonlocal Poisson brackets. Physica D, 156, Nos. 1-2, 53-80 (2001); arXiv: nlin.SI/0006030 (2000).

18. Павлов М. В. Эллиптические координаты и мультигамильтоновы структуры систем гидродинамического типа. ДАН, 339, № 1, 21-23 (1994).

19. Ферапонтов E. B. Дифференциальная геометрия нелокальных гамильтоновых операторов гидродинамического типа. Функц. анализ и его прил., 25, вып. 3, 37-49 (1991).

20. Ferapontov E. $V$. Nonlocal Hamiltonian operators of hydrodynamic type: differential geometry and applications. In: Topics in topology and mathematical physics (S. P. Novikov, ed.), Amer. Math. Soc., Providence, RI, 1995, pp. 33-58.

21. Mokhov O. I. Compatible Poisson structures of hydrodynamic type and the equations of associativity in two-dimensional topological field theory. Rep. Math. Phys., 43, No. 1/2, 247-256 (1999).

22. Гельфанд И. М., Дорфман И. Я. Гамильтоновы операторы и связанные с ними алгебраические структуры. Функц. анализ и его прил., 13, вып. 4, $13-30$ (1979).

23. Fuchssteiner B. Application of hereditary symmetries to nonlinear evolution equations. Nonlinear Anal., 3, 849-862 (1979).

24. Fokas A. S., Fuchssteiner B. On the structure of symplectic operators and hereditary symmetries. Lettere al Nuovo Cimento, 28, No. 8, 299-303 (1980).

25. Олвер П. Приложения групп Ли к дифференциальным уравнениям. Мир, М., 1989.

26. Dorfman I. Dirac structures and integrability of nonlinear evolution equations. John Wiley \& Sons, Chichester, England, 1993.

Центр нелинейных исследований при Институте теоретической физики им. Л. Д. Ландау РАН e-mail:mokhov@mi.ras.ru,mokhov@landau.ac.ru 